\title{
TRADICIÓN MODERNA E IDENTIDAD. LA ARQUITECTURA URUGUAYA EN LA SEGUNDA MITAD DEL SIGLO XX
}

Pablo Frontini 


\section{PABLO FRONTINI}

Doctor en Arquitectura, Escuela Técnica Superior de Arquitectura (ETSA), Universidad Politécnica de Cataluña (España). Homologación a título Europeo de Arquitecto, Escuela Técnica Superior de Arquitectura (ETSA), Universidad de Sevilla (España). Arquitecto, Laboratorio de Arquitectura, Escuela Técnica Superior de Arquitectura (ETSA), Universidad Politécnica de Cataluña (España), liderado por Helio Piñón y en las sedes de AH Asociados de Barcelona y Pamplona (España). Premio Nacional de Literatura de Uruguay en 2017, categoría ensayos sobre Arte.

FECHA DE RECEPCIÓN: 5 de setiembre de 2018

FECHA DE ACEPTACIÓN: 4 de diciembre de 2018.

REGISTRO BIBLIOGRÁFICO: Frontini, P. (2018). Tradición moderna e identidad. La Arquitectura Uruguaya en la Segunda Mitad del Siglo XX. Anales de Investigación en Arquitectura, 8, 7-24. 


\section{RESUMEN}

América Latina es la región en la cual, por primera vez en la historia de la arquitectura occidental, un sistema normativo de ordenación del medio físico - de máxima trascendencia como el moderno- tiene una segunda oportunidad de desarrollo, una vez agotado su recorrido dentro de su lugar de origen.

Esta constatación nos conduce a intentar comprender el proceso de síntesis transcultural que se produce mediante la adaptación de ciertas bases filosóficas, organizativas y estéticas a una realidad sustancialmente diferente a la que existía en donde fueron concebidos.

Dentro de algunas circunstancias más generales, que se intentan fundamentar de forma sucinta en el texto, se pueden observar ciertas constantes que van pautando el desarrollo de la práctica del proyecto y la producción de arquitectura moderna en el Uruguay de la segunda mitad del siglo XX. Evitando la pretensión de hacer un inventario cerrado o definitivo, parece pertinente intentar definir y sistematizar algunos principios que se reiteran, aunque en sus más diversas expresiones, tanto en la práctica arquitectónica como en los discursos de quienes han definido el perfil de los mejores ámbitos urbanos del país.

Palabras clave: Arquitectura Moderna, Uruguay, Siglo XX, Modernidad, Universalismo, Autonomía Formal, Teoría del proyecto.

\section{ABSTRACT}

Latin America is the region in which, for the first time in the history of Western architecture, a normative system for the organization of the physical environment -of maximum transcendence such as the modern one- has had a second development opportunity, once its trajectory was ended in its place of origin.

This observation leads us to try to understand the process of transcultural synthesis that occurs through the adaptation of certain philosophical, organizational and aesthetic bases to a reality substantially different from the one in which they were conceived.

Within some general circumstances that are summarized in this text, certain constants can be observed. These principles guided the production of modern architecture in Uruguay during the second half of the 20th century. Avoiding the pretension of making a closed or definitive clasification, it seems pertinent to try to define and systematize those values that can be found as constant -although in their most diverse expressions-, both in architectural practice and in theoretical explanations of those who have defined the profile of the best urban areas of the country.

Keywords: Modern Architecture, Uruguay, 20th Century, Modernity, Universalism, Formal Autonomy, Project Theory. 


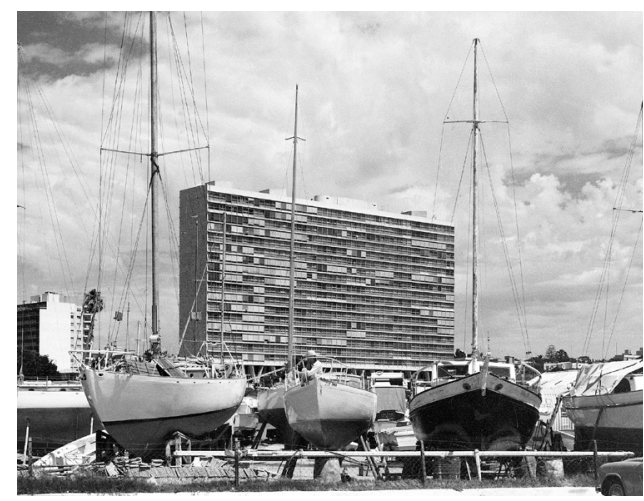

Figura 1.

Edificio Panamericano. Montevideo, 1958. Versión construida. Arq. Raúl Sichero.

Fotografía: Arq. César Loustau. Archivo de la familia Sichero.

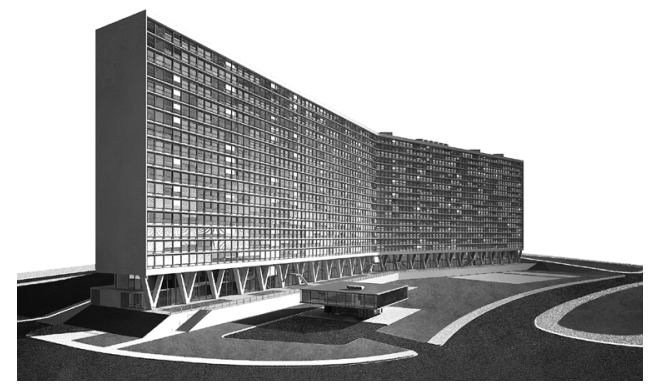

Figura 2.

Edificio Panamericano. Montevideo, 1958. Versión inicial no construida. Arq. Raúl Sichero.

Fotografía y construcción gráfica: Raúl Sichero y Pablo Frontini.

\section{TRADICIÓN MODERNA E IDENTIDAD}

América Latina es la región en la cual, por primera vez en la historia de la arquitectura occidental, un sistema de proyecto -de máxima trascendencia como el moderno- tiene una segunda oportunidad de desarrollo, una vez agotado su recorrido dentro de su lugar de origen.

Esta constatación nos conduce a intentar comprender el proceso de síntesis transcultural que se produce mediante la adaptación de principios filosóficos, organizativos y estéticos a una realidad sustancialmente diferente a la que existía en donde fueron concebidos.

Si bien otros sistemas de la importancia histórica del Barroco ya habían sido trasladados a América con éxito, su reelaboración fue casi sincrónica a su desarrollo en el sitio en que surgió. Esta reelaboración se produjo absorbiendo elementos locales de la cultura autóctona que lo convirtieron en algo nuevo, a veces demasiado alejado del original como para descifrar sus nexos con claridad. Sin embargo, la calidad de las obras barrocas realizadas en América Latina, principalmente en Brasil, en donde la combinación de elementos populares y eruditos producidos por las cofradías religiosas dio lugar a una arquitectura inédita, permitía entrever lo que sucedería con la llegada de la modernidad a América del Sur. 
Pese a que el trasvase de conocimiento desde Europa hacia América se produjo fundamentalmente mediante la conservación y reformulación de los criterios visuales que estructuraban las obras originales, en Europa a menudo se afirma que en América del Sur no se ha producido una reflexión teórica que, como mínimo, acompañara el progreso material de la modernidad americana. Esta tendría, según los críticos europeos de mayor lucidez, un desarrollo consciente a nivel visual, aunque a nivel teórico adolecía de elaboración propia.

Aunque se puede verificar la abrumadora cantidad y calidad de producción material frente a una más reducida en términos teóricos, hay autores que han desarrollado un pensamiento autónomo, propio e inédito, alcanzando a vertebrar la modernidad americana desde un punto de vista absolutamente original y arrolladoramente moderno. Es necesario en este punto nombrar al pintor uruguayo Joaquín Torres García como valedor de la autonomía moderna americana y quien ha sido referencia, no sólo en América del Sur, sino también en Europa y en Estados Unidos en donde no ha sido nunca citado por los autores que lo han utilizado de manera casi literal.

A pesar de que no ha existido un esfuerzo de independencia continuado de los sistemas culturales primogénitos, la autonomía respecto de estos es flagrante, no, como es evidente, porque se trate de una invención insólita sin fuentes de referencias conocidas, sino porque se ha mezclado con las condicionantes locales que han alterado las premisas originales. Aunque el peso del pasado ha alcanzado suficiente fuerza identificadora y estructuradora, este ha sido complementado con una dinámica de modernidad particular: la de la propia sociedad, que no se ha desarrollado con lógicas asimilables a las de los sitios de origen.

Hubo un momento en que en América del Sur la acumulación cultural interna fue capaz de proveer no sólo de material arquitectónico propio sino de una técnica eficiente para producir sus obras. Casi siempre la técnica de construcción en este continente ha sido inferior, en todos los ámbitos de comparación posibles, que la europea y más aún que la norteamericana. Sin embargo, esto no ha sido óbice para que la capacidad de sus arquitectos trascendiera estas limitaciones.

En los edificios modernos de América del Sur, las estructuras han tenido que ser más simples y de fabricación artesanal, los muros de vidrio han debido dividirse en pequeñas partes para asegurar su viabilidad económica y los revestimientos han sido menos pulidos y brillantes. No obstante, muchas veces se ha logrado disolver el límite entre interior y exterior gracias a una hábil relación de la estructura con el cerramiento. Los materiales, fueron habitualmente elegidos en virtud de su disponibilidad y sólo podían ser utilizados con medios primarios, aunque estos en diversos casos han servido más como vínculo físico 


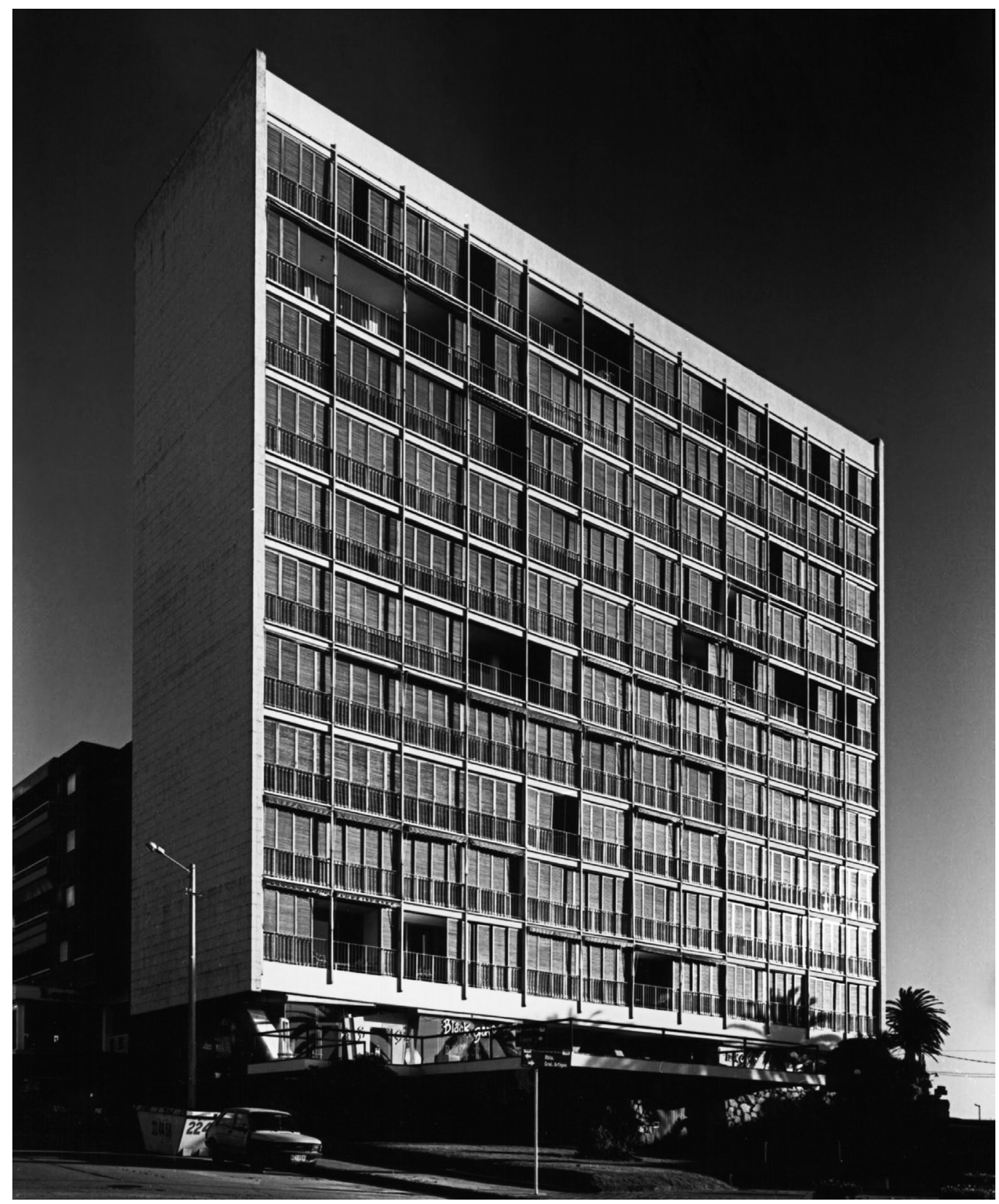

Figura 3.

Edificio Puerto. Punta del Este, 1959. Arqs. Rodolfo Lopez Rey y Gullermo Gómez Platero.

Fotografía: Archivo personal del Arq. Rodolfo López Rey.

Anales de Investigación en Arquitectura Vol.8, 2018. Montevideo (Uruguay), 7-24. 
con el lugar que como limitación insuperable. Lo necesario ha funcionado como catalizador de posibilidades más que como obstáculo, y la técnica se ha integrado de manera eficiente en la producción material como parte de la ley formal de los edificios.

Sin embargo, a pesar del gran número de arquitectos que con solvencia habían proyectado con los criterios que la modernidad puso a disposición general, sólo un puñado de ellos supo mantener sus convicciones modernas por encima de su fungibilidad general. Es justo aquí donde la arquitectura del sur de América adquiere un valor extraordinario.

\section{ESPECIFICIDAD DE LA ARQUITECTURA MODERNA EN URUGUAY}

Si bien es cierto que en Uruguay nunca existió una marcada obsesión por definir una cultura local, algunos de sus más conspicuos exponentes intentaron enunciar los postulados que vertebran su identidad. Tampoco se ha cultivado una oposición férrea a las culturas dominantes, sino que se produjo una síntesis de sus posibilidades, adaptadas al medio, con la finalidad de forjar una posición propia y original.

Otra de las particularidades de la historia del arte en Uruguay ha sido la falta de un desarrollo independiente entre teoría y práctica. Esto, que en principio parece una desventaja, no lo ha sido tanto. Quienes desarrollaban una actividad artística relevante 


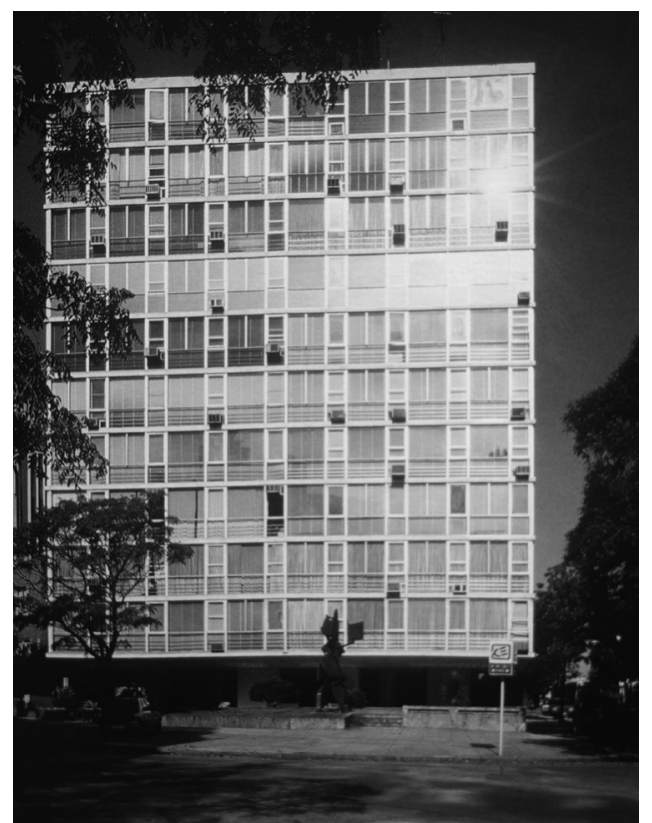

Figura 5.

Edificio Positano. Montevideo, 1959. Arq. Luis

García Pardo.

Fotografía: Arq. César Loustau. Archivo Luis García Pardo. extraían conclusiones de sus experiencias y dejaban por escrito sus observaciones acerca de unos resultados concretos, prescindiendo de conceptualizaciones ajenas a su práctica específica. Como ya se ha mencionado en el texto, el ejemplo más relevante es el de Joaquín Torres García, quien ha dejado plasmadas muchas de sus reflexiones en una amplia bibliografía. Rafael Barradas y Pedro Figari son también ejemplos significativos de pintores que trabajaron durante la primera mitad del siglo XX y dejaron constancia escrita de las reflexiones acerca de su interpretación de la modernidad y sus posibilidades de universalización.

La visión de Torres García ha logrado integrar, sin mayores contradicciones, la modernidad europea más vanguardista con el primitivismo aparente de las culturas indoamericanas, sin haber sido condicionado por las estructuras culturales existentes, por revolucionarias que hayan sido.

Esta mirada sobre una modernidad local fue, de diversas formas, continuada por los alumnos de su Escuela del Sur, pero también perpetuada por quienes, plenamente conscientes de sus valores formales, se han dedicado a prácticas más alejadas de la pintura.

En la arquitectura -seguramente la forma más pública de la producción cultural- fue aplicada buena parte del conocimiento desarrollado por Torres García en el siglo XX, mediante el cual se ha forjado la imagen física del Uruguay urbano. 
Coherencia estructural, equilibrio formal y consistencia constructiva, ligados a una determinante continuidad -permanentemente reelaborada- de la tradición artística, son sólo algunos de estos principios que defendía Torres y que, de diversos modos, caracterizaron a una gran cantidad de edificios singulares y por tanto, de espacios urbanos concebidos durante este período.

Es imposible entender al Uruguay actual sin tener en cuenta la producción de los arquitectos que construyeron durante la segunda mitad del siglo XX. Ellos forjaron la imagen de serena modernidad que tuvo el país durante buena parte del siglo pasado y que hoy paulatinamente estamos perdiendo. Durante este período se han creado o decodificado los criterios modernos que se desarrollaron en Uruguay durante su historia reciente y se han construido múltiples espacios urbanos que definen la identidad de nuestras principales ciudades. En la actualidad se nos hace muy difícil entender estos principios que surgen de una síntesis transcultural muy concreta, compleja y sofisticada, que actualmente se encuentra en un triste proceso de disolución, inmersa en una globalización, que en muchos aspectos, resulta cada vez más pobre y genérica.

Sin lugar a dudas, la construcción del Uruguay urbano ha sido un esfuerzo colectivo, catalizado por algunas buenas decisiones traducidas a normativas urbanas. Un claro ejemplo es la ley de propiedad horizontal que se pone en marcha

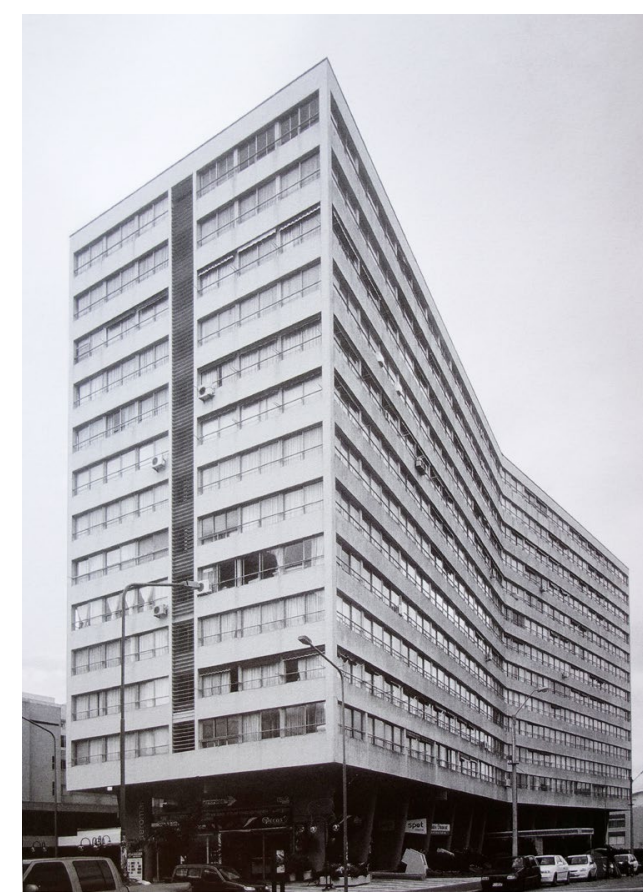

Figura 6.

Edificio Santos Dumont. Punta del Este, 1959. Arqs. Walter Pintos Risso y Raúl Sichero.

Fotografía: Arq. César Loustau. 
a fines de la década del 40, o una ordenanza trascendente que permitía volar toda la extensión del frente de los edificios en altura, admitiendo el retiro de la planta baja, para dejar expuesta la estructura del edificio.

Estableciendo una compleja relación fundamentalmente visual -pero que no excluye otra más reflexiva desde lo práctico- haciendo un uso preciso de los criterios estructuradores de la modernidad europea y norteamericana, se alcanza un resultado que en ningún caso se contrapone a sus referencias iniciales, pero que es claramente original. Esto se ha logrado mediante la combinación de los paradigmas culturales importados con los de la realidad local que, a su vez, se desarrolla dentro de los inherentes a América Latina.

Mediante la combinación reconvertida de unos pocos elementos que forman parte del extenso acervo arquitectónico moderno como la estructura, los parasoles, antepechos y barandas o las plantas bajas liberadas de masas pesadas en los rasantes-, se logra dotar de consistencia visual a varias ciudades en Uruguay. Conjugando estos elementos con la realidad geográfica y la topografía, elementos decisivos en la definición del posicionamiento y la orientación del edificio, se obtiene como resultado su posterior forma estructural. Lejos de intentar enunciar una fórmula, es necesario poner de manifiesto la complejidad de este proceso y la enorme cantidad de variantes posibles, cuya calidad no está, en absoluto, asegurada por la inclusión de estos elementos en el proyecto.

Por otra parte, el hormigón armado ha sido el material que más se ha adecuado a la realidad económica y tecnológica del país en el diseño de las estructuras portantes. En Uruguay este material no fue utilizado como alarde de los avances estructurales de vanguardia -algo que pudo haber sucedido en una fracción de la modernidad brasileña-, sino para dotar de homogeneidad, consistencia y viabilidad económica a la construcción de la enorme mayoría de los edificios domésticos e institucionales. Las obras basadas en la gestualidad de sensuales curvas, expresión de un individualismo radical, son inexistentes en el país. En cambio, se han levantado sobrios edificios, pensados con recursos técnicos y materiales similares, que han consolidado una textura urbana de alta riqueza visual, al menos en las zonas en las que se contó con los medios económicos para su desarrollo.

La estructura -a la cual Torres García tenía como principio vertebrador de su Universalismo Constructivo, y que consideraba como nexo eterno entre las genuinas obras de la antigüedad y las de vanguardia- es utilizada, con elaborada espontaneidad, por los arquitectos uruguayos, vinculándola a su realidad geográfica y adaptándola a sus condicionantes locales. Por tanto, la arquitectura moderna uruguaya del siglo XX es una expresión de madurez cultural y no de riqueza económica o tecnológica. Es 
sorprendente que esta arquitectura, de sobria creatividad y alta calidad, haya nacido en un lugar que si no se mira con cuidado podría considerarse periférico, casi marginal.

A pesar de los halagos que la arquitectura latinoamericana -en especial la brasileña y la mexicana- ha recibido en la prensa especializada de los años cuarenta y cincuenta, en términos generales, ha sido extensamente excluida de la producción de conocimiento arquitectónico más difundida.

No obstante, fue en este subcontinente luego de que la arquitectura moderna fuera desplazada de los círculos intelectuales más influyentes del mundo- en donde dicha arquitectura tuvo su espacio de desarrollo en clave universal y local, y, por tanto, original e inédita, aunque siempre transmitiendo aquel espíritu ilustrado de la Europa que la caracterizó.

Este fenómeno permitirá comparar resultados, producidos en la segunda mitad del siglo XX, entre la arquitectura del Occidente desarrollado, atacado por el despilfarro, y la menos vistosa arquitectura moderna que se realizó en algunas zonas de Latinoamérica durante el mismo período. Quizá entonces se invierta la relación entre centro y periferia, y se cierre, por fin, el círculo, al menos en términos culturales. Algo parecido ya ha pasado en el campo literario y en el musical, por lo que quizá se podría ser moderadamente optimista.

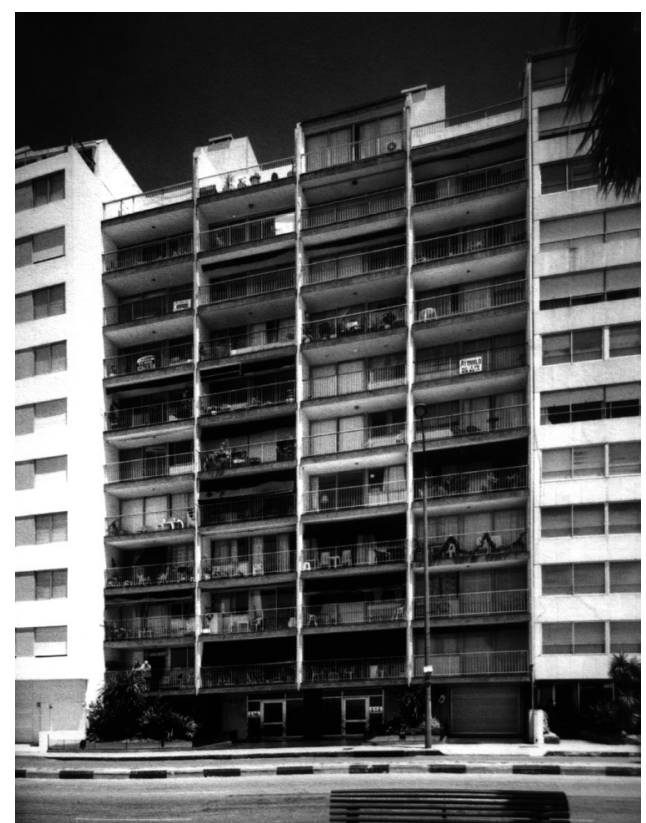

Figura 7.

Edificio Finisterre. Montevideo, 1963. Arqs Rodolfo López Rey y Guillermo Gómez Platero.

Fotografía: Arq. César Loustau. Archivo personal del Arq. Rodolfo López Rey. 


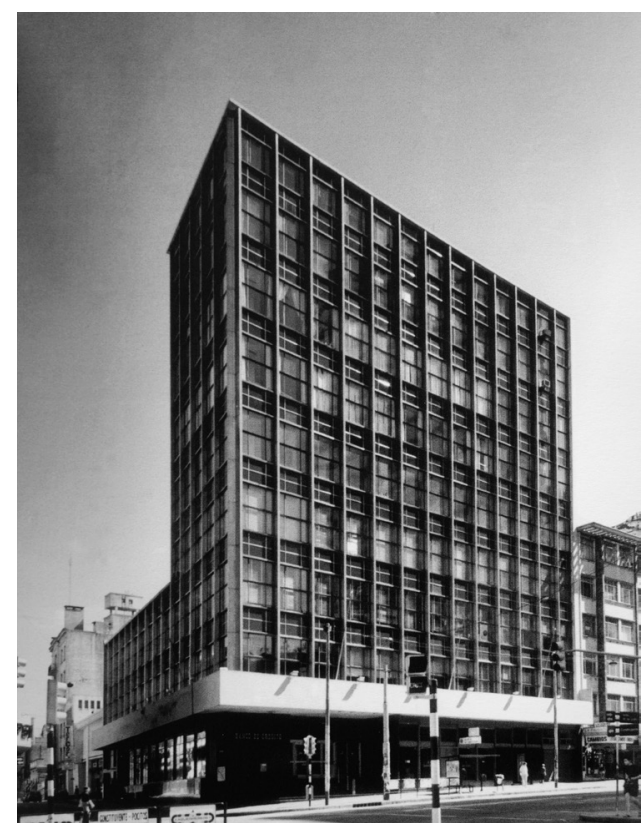

Figura 8.

Banco de Crédito. Montevideo, 1958. Arqs. Juan Antonio Rius y Luis Vaia.

Fotografía: Arq. César Loustau.

\section{CONCLUSIONES PRIMARIAS}

Dentro de estas circunstancias más generales que se intentaron fundamentar de forma sucinta en el texto precedente, se pueden observar ciertas constantes que van pautando el desarrollo de la práctica del proyecto y la producción de arquitectura moderna en el Uruguay de la segunda mitad del siglo XX. Evitando la pretensión de hacer un inventario cerrado o definitivo, parece pertinente intentar definir y sistematizar algunos principios que se reiteran, aunque en sus más diversas expresiones, tanto en la práctica arquitectónica como en los discursos de quienes han definido el perfil de los mejores ámbitos urbanos del país.

Raúl Sichero, Rodolfo Lopez Rey, Luis García Pardo, Mario Roberto Álvarez, o Miguel Amato han tenido un cometido preponderante en la construcción, padrón por padrón, de la ciudades modernas de nuestro país. Todos ellos compartieron algunos principios fundamentales sobre la práctica del proyecto, que expresaron con grados de elocuencia diversa pero con mucha claridad.

Con el riesgo de ser excesivamente esquemático y dejando múltiples conceptos por el camino, podría aventurarme a resumir cinco de los más relevantes, que por momentos están fuertemente entrelazados entre sí y que a veces se convierten en solo uno. 


\section{TRADICIÓN ARQUITECTÓNICA}

Todos ellos, de una u otra forma, han coincidido en poner en valor los pequeños y a veces imperceptibles progresos que se van logrando con el paso del tiempo, cada uno de los cuales se alza sobre los alcanzados antes. Para afrontar un nuevo proyecto arquitectónico todos coincidían, sin excepción, en aproximarse a partir de obras propias y ajenas, que consideraban con la calidad necesaria para ayudarlos a resolver los problemas a los que se enfrentaban en ese nuevo desafío.

A nadie se le ocurría empezar a proyectar a partir de un papel en blanco esperando a que alguna epifanía surgiera para socorrerlos. Trabajaban a partir de elementos constitutivos básicos que, relacionados mediante criterios de forma claros y estables, les permitían reelaborar casos previos con la ayuda de la experiencia de quienes ya habían afrontado buena parte de los problemas a resolver. Salvo cuando la prefabricación se plantea como objetivo central, ningún proyecto coincide con otro posterior como para reproducirlo sin modificaciones sustanciales. Sin embargo, una vez constatada la naturaleza y complejidades de la nueva situación que enfrentaban y, en base a juicios estéticos de carácter subjetivo que tienden a alcanzar la universalidad en cada decisión, estos arquitectos fueron poniendo su imaginación al servicio de la tradición arquitectónica moderna, no siempre con el objetivo de subvertirla, sino para intensificar sus valores más relevantes. Todos tenían incorporado a su vida profesional

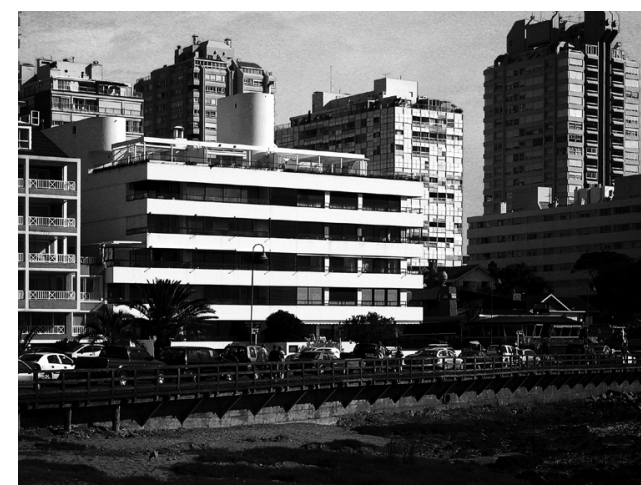

Figura 9.

Edificio Amarras del Este. Punta del Este. Arq. Raúl Sichero. Fotografía: Dr. Arq. Pablo Frontini.

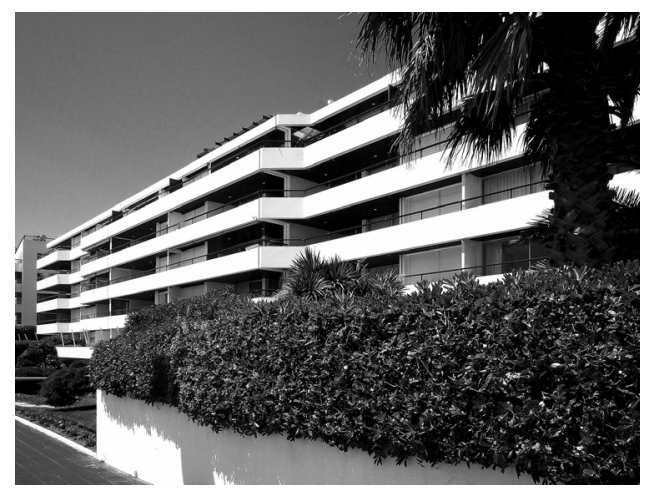

Figura 10

Edificio El Espigón. Punta del Este. Arq. Raúl Sichero. Fotografía: Dr. Arq. Pablo Frontini. 


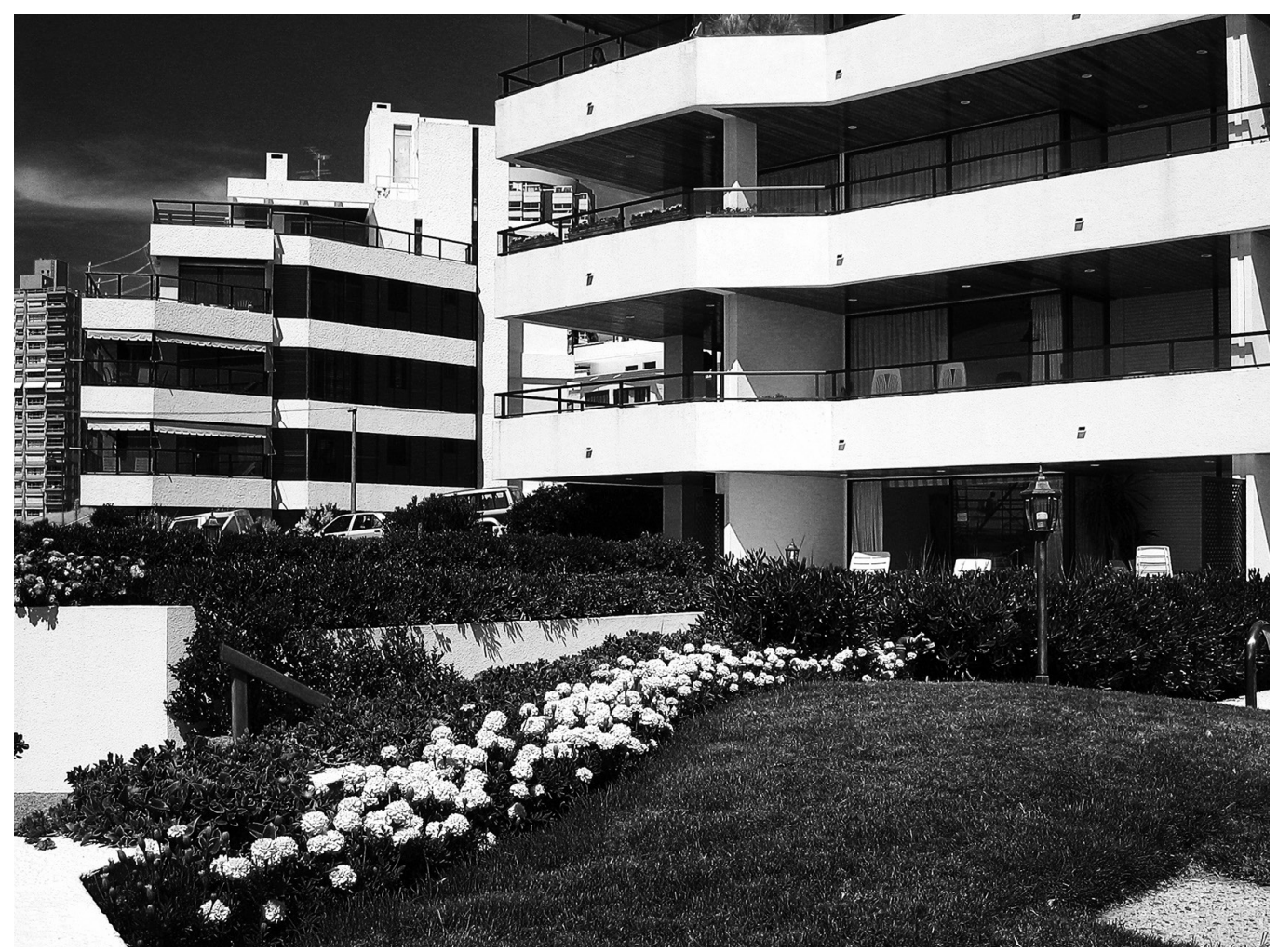

Figura 11.

Edificio Amarras del Sol del Arq. Raúl Sichero y Edificio Cigale del Arq. Walter Pintos Risso. Punta del Este Fotografía: Dr. Arq. Pablo Frontini.

el estudio sistemático de proyectos anteriores tanto clásicos como modernos- que les serían útiles para moldear sus proyectos futuros, plantear criterios de trabajo y mecanismos de proyecto generales con el objetivo de orientar su práctica arquitectónica.

La historia de la arquitectura pone a disposición de quien proyecta la materia prima necesaria para abordar los proyectos futuros. Dichos materiales, manejados con criterios de forma y responsabilidad histórica, permiten una estabilidad formal, y por tanto visual del proyecto, que no se puede alcanzar cuando se intenta producir ignorando el conocimiento y la información de calidad que nos antecede. La voluntad de continuidad con la mejor tradición arquitectónica nacional $-\mathrm{y}$ por definición internacional y moderna-, ha sido históricamente el único camino posible para aquellos arquitectos que moldearon el perfil de nuestras mejores ciudades. 


\section{LIBERTAD DE PROYECTO}

Helio Piñón suele decir que es mejor saber elegir entre dos alternativas concretas que tener mil posibilidades y no saber por cual optar. Este concepto está ligado, en parte, al arriba mencionado. No se traiciona un proyecto por el hecho de comenzar a partir del conocimiento previo sobre un problema ya estudiado -limitando en parte nuestra libertad de acción- sino que, en base a la imaginación, en combinación con la intuición y la capacidad formalizadora del arquitecto, se pueden alcanzar situaciones de proyecto de alta consistencia y complejidad que resultan imposibles de conseguir partiendo desde una situación de libertad total.

Los arquitectos mencionados no temían en absoluto el comenzar a trabajar a partir de algunas pautas dadas, orientadas a su reelaboración consciente para conseguir proyectos de alta estabilidad formal y al vez, sumamente originales. $Y$ no sólo proyectaban con las restricciones impuestas por los materiales de proyecto utilizados, sino también con las limitaciones generadas por los recursos económicos, los tecnológicos, los geográficos, climáticos y un largo etcétera.

Por tanto, la calidad de cada obra se generaba precisamente a partir de dichas condiciones, las cuales requerían una adecuación eficiente al enclave en que se insertaba, al programa funcional dado, a la técnica que se empleaba y a los materiales disponibles en el momento. Sólo a través de la mediación de la capacidad de quien proyectaba se podía llegar a trascenderlas convirtiendo al edificio en una obra de valor universal.

Dicha tensión entre una libertad individual condicionada en el proyecto por tantas razones, y la férrea voluntad de alcanzar la universalidad a cualquier costo, ha moldeado la producción de los arquitectos modernos en el país, quedando representado, lúcidamente y con gran claridad, el espíritu de una época donde se producía sincrónicamente la integración a las valores de la arquitectura moderna internacional, pero cuyas limitaciones específicas le confirieron una calidad y carácter únicos.

\section{AUTONOMIA FORMAL Y UNIDAD DE LO VISIBLE}

En la arquitectura moderna uruguaya de la segunda mitad del siglo XX los mejores arquitectos procuraron, en muchos casos, proyectar a partir de elementos con atributos formales y, por tanto estéticos, que aislados, poseen una identidad concreta. Una mirada rápida a un edificio moderno nos permite entender su volumetría general y, a la vez, identificar los principales elementos que lo componen: pilares, losas, muros, placas, lamas, tensores, varillas, etc. Al combinarse en un proyecto, sus valores individuales se intensifican dado que pasan a formar parte de un conjunto de mayor complejidad, y, sin debilitar su autonomía, trascienden su propia esencia. 
Si bien es cierto que en la mayoría de estos elementos se repiten en cada proyecto, la forma de combinarlos hace que cada obra sea única e irrepetible, siempre que las condicionantes de su enclave determinen su geometría base y la configuración de su estructura.

Por un lado, la arquitectura moderna ha cimentado buena parte de sus avances técnicos y formales en la configuración de sistemas repetibles que atiendan al carácter industrializado de su producción en relación a la época a la que pertenecen. Por otro, un sistema de componentes basados en un módulo aditivo e intercambiable para encajar en cualquier situación, no ofrece las garantías esenciales de calidad arquitectónica: el carácter de la forma del edificio se altera de acuerdo al tamaño del mismo, su situación y programa, determinando la transformación del sistema para su necesaria adaptación al proyecto. Es la voluntad de forma y la sensibilidad propia de cada proyectista que, al combinarlos con precisión, termina definiendo el valor arquitectónico de cada intervención.

Es por esto que la unidad formal del tejido construido en esa época del país -conformado por obras proyectadas a lo largo de medio siglo y en circunstancias tan complejas como diversas-, se debe a una búsqueda consciente de la unidad de lo visible y de una universalidad arquetípica de los proyectos desde la autonomía de su forma. Inevitablemente cada arquitecto partía de su interpretación subjetiva de la realidad, aunque sin renunciar al origen de los principios, criterios y soluciones que enmarcaban la formalidad concreta de cada edificio. Tal como dice Helio Piñón, la identidad de cada obra se basa en el encuentro de la sistematicidad que la herencia propicia y la singularidad de las condiciones en que emerge cada proyecto. Dicha identidad es lo que construye e intensifica la unidad del entorno urbano a medida que se va incorporando cada nueva obra, trascendiendo su autoría y franja temporal concreta.

\section{NOCIONES DISCIPLINARES ESPECIFICAS}

Otro tema fundamental que se reiteraba en las conversaciones con Raúl Sichero y en el presente de forma constante con Rodolfo López Rey, es el abordaje del proyecto arquitectónico desde las nociones propias y esenciales de la arquitectura. El carácter intelectual y sensible de la forma arquitectónica surge siempre a partir de temas específicos de la disciplina y su sentido intrínseco. La estructura, la técnica, la escala, la construcción -y demás temas inmanentes a la arquitectura- funcionaron como catalizadores y fueron los fundamentos, las herramientas y la finalidad de los proyectos de estos importantes arquitectos.

Además, la actividad de proyectar, en tanto se encuentra dentro de la esfera del arte, se aleja de todo conceptualismo en términos discursivos y está, por otra parte, en estrecha relación con la visualidad. De acuerdo con Helio Piñón "el juicio, proceso esencial de la 
experiencia estética, se produce -como Kant señaló hace más de dos siglos- por medio de una interacción atípica de los sentidos y el entendimiento. No se trata, por tanto, de escoger entre la razón o los sentidos, ya que estos por sí mismos, sin el concurso de aquella, no provocan más que meras sensaciones, en las que no es posible ningún reconocimiento de forma."

La capacidad de juicio estético sucede por una influencia recíproca entre la intelección visual y la razón, y en el cual el conocimiento profundo del marco cultural en el cual se inscribe tiene un peso determinante.

El necesario carácter tectónico de los edificios los aleja también de las finalidades decorativas, pasando a ser estás parte del orden constructivo -mediado por la capacidad formadora del arquitecto- del edificio. La singularidad funcional, técnica o de situación que estimuló la obra queda integrada en su sistematicidad global y el control de la apariencia de sus componentes, realizado por el arquitecto, ha trascendido el mero sistema de construcción.

Su calidad plástica se obtiene de la tensión formal aplicada a los materiales -a sus dimensiones y vínculos-, a los elementos que conforman el proyecto y al conjunto de relaciones entre ellos que interviene en la composición.

\section{UNIVERSALIDAD}

Fuertemente ligado a los conceptos anteriores, existe uno que los sobrevuela a todos y es el de arquetipo formal, entendido como un modelo que sirve como pauta para ser imitado, reproducido o copiado. Esta idea que ha permitido, durante más de dos mil años, generar un conocimiento acumulativo en arquitectura $-y$ que sólo se ha abandonado hace poco más de cuatro décadas- es la que ha permitido a la arquitectura entrar dentro del ámbito del arte y por tanto conferirle un carácter de universalidad.

Tal como lo hemos indicado previamente, este concepto está presente en las raíces más profundas de la producción de los arquitectos modernos que hemos mencionado. Todos ellos partían de edificios existentes que consideraban de máxima calidad como referencia previa de su propia actividad con el fin de calibrar la estabilidad formal de sus propios proyectos.

Todos consideraban que la existencia de estos arquetipos generales representaban y contenían, en su esencia formal, lo necesario para resolver las situaciones particulares que la realidad de su proyecto les presentaba.

Es así que consideraban como punto de partida o material de proyecto ejemplos paradigmáticos del pasado y simultáneamente priorizaban la continuidad de sus propuestas previas hasta lograr la mejor versión posible de sus propios proyectos. De esta manera no sólo iban creando 
soluciones constructivas y formales cada vez más eficientes sino que iban generando sectores urbanos de gran consistencia y calidad.

Sus propuestas formales, aunque intensamente teñidas por sus interpretaciones subjetivas de las dimensiones tectónicas del proyecto, permanecían inmersas dentro de las convenciones de la mejor tradición arquitectónica y dentro de unas dimensiones históricas que los trascendían a sí mismos y a su época. La concepción de sus proyectos en base a abstracciones de arquetipos formales hizo posible, a través de la sutil interpretación sensible de cada arquitecto, la revelación de una forma radicalmente original y, a la vez, indudablemente universal.

La mayoría de las ciudades del Uruguay cuentan con espacios urbanos o frentes costeros modernos caracterizados por los principios arriba mencionados, los cuales vertebran, al menos en parte, la forma concreta que estos arquitectos tienen o tenían de entender la arquitectura. No solo debemos ser conscientes de su valor estético -y por tanto arquitectónico y urbanístico- sino que debemos encontrar los medios adecuados para lograr su preservación para las futuras generaciones.

\section{BIBLIOGRAFÍA}

ADORNO, Th. W. (1970). Teoría estética. Madrid: Akal, 2004

FRONTINI, P. (2015). Raúl Sichero. Arquitectura Moderna y Calidad Urbana. Facultad de Arquitectura. Udelar

GASTÓN, C. (2005). Mies: el proyecto como revelación del lugar. Barcelona: Arquithesis.

GREENBERG, C. (1961). Art and culture. Boston, Massachusetts: Beacon Press.

HILDEBRAND, A. (1893). El problema de la forma en la obra de arte. Madrid: La Balsa de la Medusa, 1988.

ORTEGA Y GASSET, J. (1925). La dehumanización del arte. Barcelona: Espasa Libros, 2003.

PIÑÓN, H. (2002). Raúl Sichero. Barcelona: Edicions UPC, ETSAB.

PIÑóN, H. (2006). Teoría del proyecto. Barcelona: Edicions UPC, ETSAB.

ROWE, C. (1999). Manierismo y arquitectura moderna y otros ensayos. Barcelona: GG Reprints.

TORRES GARCÍA, J. (1944). Universalismo constructivo. Buenos Aires: Poseidón. 